\title{
Second Derivatives of Circle Packings and Conformal Mappings
}

\author{
Peter Doyle, ${ }^{1}$ Zheng-Xu He, ${ }^{1}$ and Burt Rodin ${ }^{2}$ \\ ${ }^{1}$ Princeton University, Princeton, NJ 08544, USA \\ ${ }^{2}$ Department of Mathematics, University of California, \\ San Diego, La Jolla, CA 92093-0112, USA
}

\begin{abstract}
William Thurston conjectured that the Riemann mapping function $f$ from a simply connected region $\Omega$ onto the unit disk $\mathbb{D}$ can be approximated as follows. Almost fill $\Omega$ with circles of radius $\varepsilon$ packed in the regular hexagonal pattern. There is a combinatorially isomorphic packing of circles in $\mathbb{D}$. The correspondence $f_{\varepsilon}$ of $\varepsilon$-circles in $\Omega$ with circles of varying radii in $\mathbb{D}$ should converge to $f$ after suitable normalization. This was proved in [RS], and in [H] an estimate was obtained which led to an approximation of $\left|f^{\prime}\right|$ in terms of $f_{\varepsilon}$; namely, $\left|f^{\prime}\right|$ is the limit of the ratio of the radii of a target circle of $f_{\varepsilon}$ to its source circle. In the present paper we show how to approximate $f^{\prime}$ and $f^{\prime \prime}$ in terms of $f_{\varepsilon}$. Explicit rates for the convergence to $f, f^{\prime}$, and $f^{\prime \prime}$ are obtained. In the special case of convergence to $\left|f^{\prime}\right|$, the estimate in this paper improves the previously known estimate.
\end{abstract}

\section{Introduction}

In [T] Thurston conjectured that the Riemann mapping function from a region $\Omega$ onto the unit disk $\mathbb{D}$ can be approximated as follows. Almost fill $\Omega$ with circles of radius $\varepsilon$ packed in the regular hexagonal pattern. There is a combinatorially isomorphic packing of circles in $\mathbb{D}$. This correspondence $f_{\varepsilon}$ of $\varepsilon$-circles in $\Omega$ with circles of varying radii in $\mathbb{D}$ should converge, with suitable normalizations, to the Riemann map $f: \Omega \rightarrow \mathbb{D}$.

This conjecture was proved in [RS]. There the question was raised of whether the ratio of the radius of a target circle to the radius of its source circle under $f_{\varepsilon}$ converges to $\left|f^{\prime}\right|$. In $[\mathrm{H}]$ an estimate was obtained which gave an affirmative answer to this question. The estimate also provided, in conjunction with [R2], an explicit estimate for the rate of convergence $f_{\varepsilon} \rightarrow f$. 
In this paper we approach the problem of approximating $f^{\prime}$ and $f^{\prime \prime}$ in terms of $f_{\varepsilon}$ as follows. A triple of mutually tangent $\varepsilon$-circles in the packing of $\Omega$ corresponds under $f_{\varepsilon}$ to a triple of mutually tangent circles in the packing of $\mathbb{D}$. There is a unique Möbius transformation which maps the first triple onto the second. The first and second derivatives of $f_{\varepsilon}$ in the neighborhood of the three source circles are defined to be the derivatives of this Möbius transformation.

With this definition we show that $f_{\varepsilon}^{\prime} \rightarrow f^{\prime}$ and $f_{\varepsilon}^{\prime \prime} \rightarrow f^{\prime \prime}$ (this method does not work for higher-order derivatives since the Schwarzian derivative of $f$ need not vanish). The method gives explicit estimates for the rates of convergence of $f_{\varepsilon} \rightarrow f$, $f_{\varepsilon}^{\prime} \rightarrow f^{\prime \prime}$; see Theorem 2.4. The rate which is obtained for $f_{\varepsilon} \rightarrow f$ is an improvement over the previously known estimate.

In this paper we treat only circle packings having the hexagonal pattern. Similar results but with weaker estimates can be obtained for other classes of circle packings by combining the techniques of this paper with those in [HR].

\section{Local Theory}

In this section we present results from [H] and derive consequences which are needed later. We consider a base circle and let $H_{n}$ denote the regular hexagonal circle packing of $n$ generations around $c_{0}$; by "regular" we mean that all circles have the same radius. Let $H_{n}^{\prime}$ denote a circle packing which has the same tangency combinatorics as $H_{n}$; the circles of $H_{n}^{\prime}$ need not have equal radii. Let $c \rightarrow c^{\prime}$ denote the correspondence of circles under the combinatorial isomorphism $H_{n} \rightarrow H_{n}^{\prime}$.

A circle $\tilde{c}$ (resp. $\tilde{c}^{\prime}$ ) which passes through the three points of tangency of three mutually tangent circles of $H_{n}$ (resp. $H_{n}^{\prime}$ ) is called a dual circle of $H_{n}$ (resp. $H_{n}^{\prime}$ ), and the disk $\tilde{D}$ (resp. $\tilde{D}^{\prime}$ ) which it bounds is called a dual disk of $H_{n}$ (resp. $H_{n}^{\prime}$ ); see Fig. 1.

Notation. Let $c_{i}, c_{j}, c_{k}$ be three mutually tangent circles of $H_{n}$, and let $\tilde{D}$ be the dual disk determined by the three tangency points $c_{i} \cap c_{j}, c_{j} \cap c_{k}, c_{k} \cap c_{i}$. The Möbius transformation which sends $c_{i} \cap c_{j}, c_{j} \cap c_{k}, c_{k} \cap c_{i}$ to the corresponding tangency points $c_{i}^{\prime} \cap c_{j}^{\prime}, c_{j}^{\prime} \cap c_{k}^{\prime}, c_{k}^{\prime} \cap c_{i}^{\prime}$ in $H_{n}^{\prime}$ is denoted by $M_{\tilde{D}}$.

Throughout this paper $C, C_{1}, C_{2}, \ldots$ denote absolute positive constants which may change with the context.

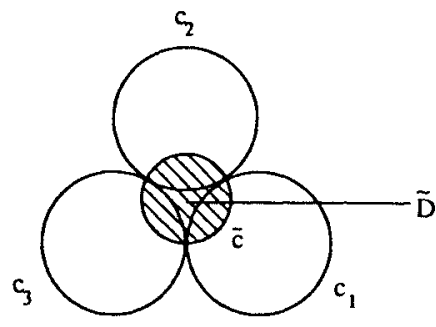

Fig. 1. $c_{1}, c_{2}, c_{3}$ are mutually tangent circles of $H_{m}$ and $\tilde{c}$ is the circle dual to $c_{1}, c_{2}, c_{3}$. The shaded disk $\tilde{D}$ is a dual disk of $H_{m}$. 


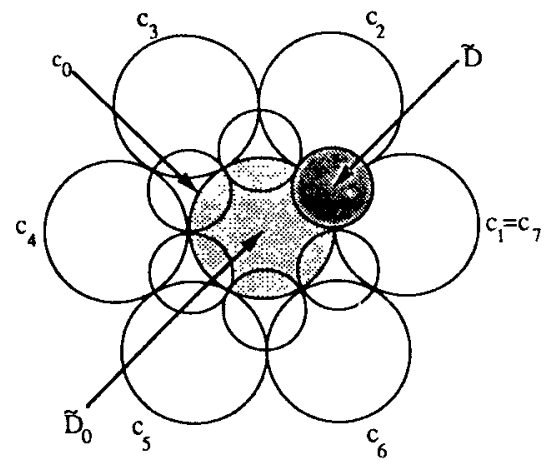

Fig. 2.

Lemma 1.1. Given $H_{n}$ and $H_{n}^{\prime}$, where $n \geq 2$, there is a C-quasi-conformal homeomorphism $\mathrm{g}: \mathbb{C} \rightarrow \mathbb{C}$ with the following properties:

(i) $g$ maps $c_{0}$ and its six neighbors $c_{i}(1 \leq i \leq 6)$ onto the corresponding circles $c_{i}^{\prime}(0 \leq i \leq 6)$ of $H_{n}^{\prime}$, and maps the center of $c_{0}$ to the center of $c_{0}^{\prime}$.

(ii) Let $\tilde{D}$ be a dual disk of $H_{n}$ which intersects $c_{0}$. Then $\left.g\right|_{\tilde{D}}$ is equal to the restriction to $\tilde{D}$ of the Möbius transformation $M_{\tilde{D}}$ (see Fig. 2).

(iii) Let $D_{0}$ be the disk bounded by $c_{0}$. Then the $\left.g\right|_{D_{0}}$ is conformal except on $a$ "bad" subset of area $\leq\left(C / n^{2}\right) \operatorname{Area}\left(D_{0}\right)$.

Proof. A detailed proof is given in [H]. Briefly, $g: \mathbb{C} \rightarrow \mathbb{C}$ can be constructed as follows. First define $g$ on each triangular interstice of $H_{n}$ to be the unique Möbius transformation which maps that interstice onto the corresponding interstice of $H_{n}^{\prime}$. This defines $g$ on each circle of $H_{n}$. The Ring Lemma of [RS] shows that the radial extension to the inside of each circle will be quasi-conformal. Redefine $g$ outside the circles and interstices of $H_{[n / 2]}$ to obtain a $C$-quasi-conformal homeomorphism of the entire plane. Finally, modify this map on the inside of the circles so that it becomes equivariant with respect to inversions in the circles of $H_{n}$. Since inversions of interstices pave the dual circles, conditions (ii) will be satisfied. The proof of condition (iii) requires delicate estimates of the area of the images of dual circles under inversions.

Lemma 1.2. Given $H_{n}$ and $H_{n}^{\prime}$, where $n \geq 2$, let $P_{n-1}$ (resp. $P_{n-1}^{\prime}$ ) be the polygon whose boundary consists of line segments joining the centers of pairs of tangent circles of generation $n-1$ of $H_{n}\left(\right.$ resp. $\left.H_{n}^{\prime}\right)$; see Fig. 3. Then there is a $C$-quasi-conformal homeomorphism $g_{n}: P_{n-1} \rightarrow P_{n-1}^{\prime}$ with the following properties:

(i) $g_{n}$ maps circles or arcs of circles of $H_{n-1}$ onto the corresponding circles or arcs of circles of $H_{n-1}^{\prime}$.

(ii) For each dual disk $\tilde{D}$ of $H_{n-1},\left.g_{n}\right|_{\tilde{D}}=\left.M_{\tilde{D}}\right|_{\tilde{D}}$, where $M_{\tilde{D}}$ is the Möbius transformation defined above.

(iii) For any disk $D$ in $H_{k} \backslash H_{k-1}$, where $0 \leq k \leq n-2$ (convention: $H_{-1}=\varnothing$ ), 


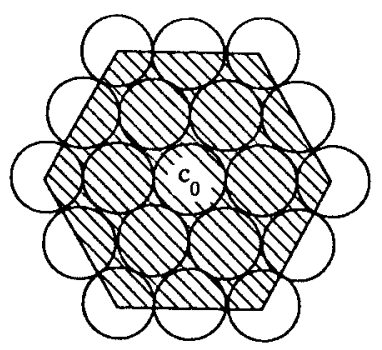

Fig. 3. $\quad P_{2}=$ the shaded polygon.

the area of the subset of $D$ where $g_{n}$ fails to be conformal is bounded from above by

$$
\frac{C}{(n-k)^{2}} \operatorname{Area}(D)
$$

Proof. First define $g_{n}$ in each dual disk $\tilde{D}$ of $H_{n}$ by $\left.g_{n}\right|_{\tilde{D}}=\left.M_{\tilde{D}}\right|_{\tilde{D}}$. Conditions (i) and (ii) will then be satisfied.

Consider a circle $c \in H_{k} \backslash H_{k-1}(0 \leq k \leq n-2)$ and let $D$ be the disk it bounds. By Lemma 1.1 there is a $C$-quasi-conformal mapping $g$ from $D$ to the corresponding disk $D^{\prime}$ in $H_{n}^{\prime}$ such that:

(a) on each dual disk $\tilde{D}$ of $H_{n},\left.g\right|_{D \cap \tilde{D}}=\left.g_{n}\right|_{D \cap \tilde{D}}$, and

(b) the area of the subset of $D$ on which $g$ fails to be conformal is less than $\left(C /(n-k)^{2}\right) \operatorname{Area}(D)$.

By defining $\left.g_{n}\right|_{D}=g$ we obtain a $C$-quasi-conformal map $g_{n}$ defined on the union of disks and dual disks of $H_{n-1}$ which satisfies properties (i)-(iii). The complement in $P_{n-1}$ of this union consists of regions such as those shown in Fig. 4.

It remains to extend $g_{n}$ uniformly quasi-conformally to these regions so the images are the corresponding regions in $P_{n-1}^{\prime}$. All the circles $c, c_{1}, c_{2}, \ldots$ in Fig. 4 are in $H_{n-1}$. By the Ring Lemma of [RS], the radii of the corresponding circles in $H_{n}^{\prime}$ have uniformly bounded ratios. Thus it is elementary to extend $g_{n}$ suitably.

Lemma 1.3. The map $g_{n}: P_{n-1} \rightarrow P_{n-1}^{\prime}$ of Lemma 1.2 is conformal except for $a$ subset whose area is bounded from above by $(C / n) \operatorname{Area}\left(P_{n-1}\right)$.

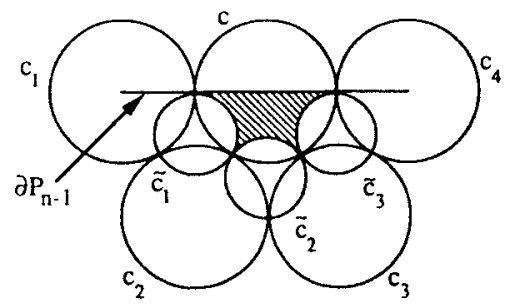

(a)

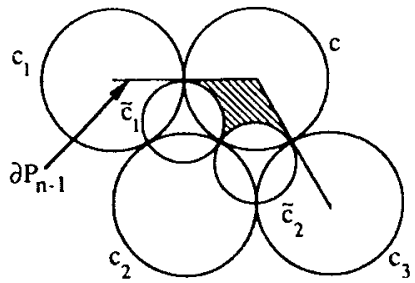

(b)

Fig. 4. (a) $c_{1}, c, c_{4} \in H_{n-1} \backslash H_{n-2}, c_{2}, c_{3} \in H_{n-2}$, and $\tilde{c}_{1}, \tilde{c}_{2}, \tilde{c}_{3}$ are dual circles. (b) $c_{1}, c_{3} \in H_{n-1} \backslash H_{n-2}$, $c_{2} \in H_{n m 2}$, and $\tilde{c}_{1}, \tilde{c}_{2}$ are dual circles. 
Proof. The bad subset of $g_{n}$ is contained in the union of disks bounded by the circles of $H_{n-1}$. Let $r$ be the radius of the circles of $H_{n}$. The number of circles in $H_{k} \backslash H_{k-1}$ is $6 k$ (or 1 if $k=0$ ). For $0 \leq k \leq n-2$, Lemma 1.2 implies that the bad area inside the circles of $H_{k} \backslash H_{k-1}$ is bounded above by $\left(6 k C /(n-k)^{2}\right) \pi r^{2}$ (or $\left(C / n^{2}\right) \pi r^{2}$ if $\left.k=0\right)$. This bound also applies for $k=n-1$ if we let $C \geq 1$. Thus the total bad area is bounded by

$$
\begin{aligned}
\frac{C \pi r^{2}}{n^{2}}+\sum_{k=1}^{n-1} \frac{6 k}{(n-k)^{2}} C \pi r^{2} & =\frac{C \pi r^{2}}{n^{2}}+\sum_{j=1}^{n-1} \frac{6(n-j)}{j^{2}} C \pi r^{2} \\
& \leq \frac{C \pi r^{2}}{n^{2}}+6 C n \pi r^{2} \sum_{j=1}^{n-1} \frac{1}{j^{2}} \\
& \leq C_{1} n r^{2}=\frac{C_{2}}{n} \operatorname{Area}\left(P_{n-1}\right)
\end{aligned}
$$

If a quasi-conformal self-map $\varphi$ of the unit disk satisfies $\varphi(0)=0, \varphi(1)=1$, and is conformal except for a set of measure $\varepsilon$, then $|\varphi(z)-z|<C \sqrt{\varepsilon}$; this fact is referred to as the Grötsch argument for conformal moduli and was proved in $[\mathrm{H}]$ as an essential step in the $C / n$-estimate for $s_{n}$. Together with the proof of Lemma 1.2 it gives

Lemma 1.4. Let $g_{n}: P_{n-1} \rightarrow P_{n-1}^{\prime}$ be as in Lemma 1.2. Then there is a euclidean similitude $S$ (that is, a map of the form $z \rightarrow S(z)=z_{0}^{\prime}+r e^{i \theta}\left(z-z_{0}\right)$ where $z_{0}$ and $z_{0}^{\prime}$ are the centers of $c_{0}$ and $c_{0}^{\prime}$ ) of the inside of $c_{0}$ onto the inside of $c_{0}^{\prime}$ such that, for all $z$ inside $c_{0}$,

$$
\left|g_{n}(z)-S(z)\right|<\left(\frac{C}{n}\right) \operatorname{Radius}\left(c_{0}^{\prime}\right)
$$

The following variation of Lemma 1.4 allows a better approximation for $g_{n}$ in some circumstances.

Lemma 1.5. Let $g_{n}: P_{n-1} \rightarrow P_{n-1}^{\prime}$ be as in Lemma 1.2. Let $D$ be a disk of $H_{k}(k \leq n-2)$ and let $\tilde{D}$ be a dual disk of $H_{n-1}$ which meets $D$. Then

$$
g_{n}(D)=M_{D}(D)=D^{\prime}
$$

where $M_{\tilde{D}}$ is the Möbius transformation which was defined above Lemma 1.1 and $D^{\prime}$ is the disk of $H_{n-1}^{\prime}$ which corresponds to $D$, and

$$
\left|g_{n}(z)-M_{\mathscr{D}}(z)\right| \leq \frac{C}{(n-k)^{2}} \operatorname{Radius}\left(D^{\prime}\right), \quad \forall z \in \partial D
$$




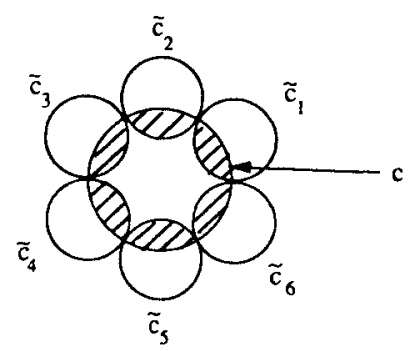

Fig. 5. $\bar{D} \cap \tilde{D}_{i}(1 \leq i \leq 6)$ are the shaded regions.

Proof. The fact that $g_{n}(D)=M_{\tilde{D}}(D)=D^{\prime}$ follows from Lemma 1.2(i). We may assume that $\operatorname{Radius}(D)=\operatorname{Radius}\left(D^{\prime}\right)=1$. Now $M_{\tilde{D}}$ maps $\tilde{D}$ to $\tilde{D}^{\prime}$ and carries the three circles of $H_{n-1}$ which determine $\tilde{D}$ - say $c_{i}, c_{j}, c_{k}$-to three circles $c_{i}^{\prime}, c_{j}^{\prime}, c_{k}^{\prime}$ of $H_{n-1}^{\prime}$. One of these three circles from $H_{n-1}$ is $\partial D$. By the Ring Lemma [RS] and the normalization $\operatorname{Radius}(D)=\operatorname{Radius}\left(D^{\prime}\right)=1$, we conclude that the radii of $c_{i}^{\prime}, c_{j}^{\prime}, c_{k}^{\prime}$ are all uniformly bounded from below. Hence $\left|(d / d z) M_{\tilde{D}}\right|$ is uniformly bounded above in $D$ and therefore $\left|g_{n}(z)-M_{D}(z)\right| \leq C_{1}\left|M_{\bar{D}}^{1} \circ g_{n}(z)-z\right|$. Hence it suffices to prove

$$
\left|M_{\bar{D}}^{-1} \circ g_{n}(z)-z\right| \leq C(n-k)^{-2}, \quad \forall z \in \partial D
$$

Let $\tilde{D}_{1}=\tilde{D}, \tilde{D}_{2}, \ldots, \tilde{D}_{6}$, be the six dual disks of $H_{n-1}$ which meet $D$ (see Fig. 5). By Lemma 1.2(ii), the restriction of $h \equiv M_{\bar{D}}^{-1} \circ g_{n}: \bar{D} \rightarrow \bar{D}$ to each region $\bar{D} \cap \widetilde{D}_{i}$, $1 \leq i \leq 6$, is a Möbius transformation. In particular, the bad subset $B \subset D$ where $h$ (equivalently, $g_{n}$ ) fails to be conformal satisfies

$$
B \subset D \backslash \bigcup\left\{D \cap \tilde{D}_{i}: 1 \leq i \leq 6\right\}
$$

and

$$
\operatorname{Area}(B) \leq C(n-k)^{-2}
$$

Since the restriction of $h$ to $D \cap \tilde{D}_{1}$ is the identity, (1.3) will follow if we show that

$$
|h(z)-z| \leq C(n-k)^{-2}, \quad \forall z \in \partial D \cap \tilde{D}_{i} \text { for } i=2, \ldots, 6
$$

Since $h$ is a Möbius transformation on $D \cap \tilde{D}_{i}$, it suffices to prove (1.5) only for three distinct points $\zeta$ on the interior of the arc $\partial D \cap \tilde{D}_{i}$ (for $i=2, \ldots, 6$ ).

Fix three points $w_{1}, w_{2}, w_{3}$ on the interior of the $\operatorname{arc} \partial D \cap \tilde{D}_{1}$. They will be left fixed by $h$. Consider $\zeta$ on the interior of the $\operatorname{arc} \partial D \cap \tilde{D}_{i}(i \neq 1)$. The quadrilaterial $\left(\bar{D}, w_{1}, w_{2}, w_{3}, \zeta\right)$ is mapped by $h$ onto the quadrilateral $\left(\bar{D}, w_{1}, w_{2}, w_{3}, h(\zeta)\right)$. Since the bad set $B$ is bounded away from $w_{1}, w_{2}, w_{3}, \zeta$ (see (1.4a)), a Grötsch argument similar to [H] using (1.4b) yields an estimate on the 
moduli of these quadrilaterals

$$
\left|\bmod \left(\bar{D}, w_{1}, w_{2}, w_{3}, \zeta\right)-\bmod \left(\bar{D}, w_{1}, w_{2}, w_{3}, h(\zeta)\right)\right| \leq C_{1}(n-k)^{-2}
$$

and consequently the desired estimate $|h(\zeta)-\zeta| \leq C_{2}(n-k)^{-2}$.

Lemma 1.6. Let $g_{n}: P_{n-1} \rightarrow P_{n-1}^{\prime}$ be as in Lemma 1.2, let $k<n-1$, let $c$ be a circle in $H_{k}$, and let $c_{0}$ be the base circle in $H_{0}$. Then

$$
\frac{1}{C^{k /(n-k)}} \leq \frac{\operatorname{Radius}\left(g_{n}(c)\right)}{\operatorname{Radius}\left(g_{n}\left(c_{0}\right)\right)} \leq C^{k /(n-k)}
$$

Proof. The estimate $s_{N} \leq C_{1} / N$ in [H] implies that if $c^{\prime}$ and $c^{\prime \prime}$ are tangent circles in $H_{n-1}^{\prime}$ of generation $<k$, then

$$
1-\frac{C_{1}}{(n-k)} \leq \frac{\operatorname{Radius}\left(c^{\prime}\right)}{\operatorname{Radius}\left(c^{\prime \prime}\right)} \leq 1+\frac{C_{1}}{(n-k)}
$$

Let $c_{0}, c_{1}, \ldots, c$ be a chain of tangent circles in $H_{n-1}$ of generations $0,1, \ldots$ up to the generation ( $\leq k)$ of $c$. Apply (1.7) to adjacent pairs in $g_{n}\left(c_{0}\right), g_{n}\left(c_{1}\right), \ldots, g_{n}(c)$ and multiply to get

$$
\frac{1}{C^{k /(n-k)}} \leq\left(1-\frac{C_{1}}{(n-k)}\right)^{k} \leq \frac{\operatorname{Radius}\left(g_{n}(c)\right)}{\operatorname{Radius}\left(g_{n}\left(c_{0}\right)\right)} \leq\left(1+\frac{C_{1}}{(n-k)}\right)^{k} \leq C^{k /(n-k)}
$$

It will be convenient to normalize the scale factor so that $P_{n}$ is a regular hexagon of side length $L$, where $L$ remains within prescribed bounds $\kappa^{-1} \leq L \leq \kappa . O_{\kappa}(x)$ and $O_{\kappa, r}(x)$ denote functions such that $\left|O_{\kappa}(x) / x\right|$ is bounded by a constant depending only on $\kappa$, and $\left|O_{\kappa, t}(x) / x\right|$ is bounded by a constant which depends on $\kappa$ and $t$. The bounds may change from one occurrence to the next. Constants denoted $C_{\kappa}$ (or $C_{\kappa, t}$ ) depend on $\kappa$ (or $\kappa$ and $t$ ).

The following corollary follows from Lemmas $1.4-1.6$ by taking $c_{0}$ and $c_{0}^{\prime}$ to have radii $O_{\kappa}(1 / n)$. Part (iv) is an immediate consequence of part (ii).

Corollary 1.7. Let $\kappa$ be a fixed positive constant. Normalize the radii of the circles in $H_{n}$ and $H_{n}^{\prime}$ so that $\kappa / 2 n \leq \operatorname{Radius}\left(c_{0}\right) \leq(2 \kappa n)^{-1}$ and

$$
\kappa / 2 n \leq \operatorname{Radius}\left(c_{0}^{\prime}\right) \leq(2 \kappa n)^{-1}
$$

For $z \in P_{n-1}$ let $M_{z}$ denote the Möbius transformation $M_{\bar{D}}$ (see the definition preceding Lemma 1.2), where $\tilde{D}$ is a dual disk of $H_{n}$ closest to $z$. Let $t>0$ and let 
$P(t)=\left\{z \in P_{n-1}: \operatorname{Dist}\left(z, \partial P_{n-1}\right) \geq t\right\}$. Let $g_{n}: P_{n-1} \rightarrow P_{n-1}^{\prime}$ be as in Lemma 1.2:

(i) For any disk $D$ in $H_{n-2}$ which meets $P(t)$ there is a euclidean similitude $S$ of $D$ onto $D^{\prime}$ such that

$$
g_{n}(z)-S(z)=O_{\kappa, t}\left(\frac{1}{n^{2}}\right), \quad \forall z \in \tilde{D} .
$$

(ii) For any disk $D$ in $H_{n-2}$ which meets $P(t)$ and for any $z_{0} \in \tilde{D}$ we have

$$
g_{n}(z)-M_{z_{0}}(z)=O_{\kappa, 1}\left(\frac{1}{n^{3}}\right), \quad \forall z \in \partial D
$$

(ii') For any disk $D$ in $H_{n-2}$ which meets $P(t)$ and for any $z_{0} \in \tilde{D}$ we have

$$
g_{n}(z)-M_{z_{0}}(z)=O_{\kappa, t}\left(\frac{1}{n^{2}}\right), \quad \forall z \in D
$$

(iii) For any disk $D$ in $H_{n-1}$ which meets $P(t)$ we have

$$
\operatorname{Radius}\left(g_{n}(D)\right)=O_{\kappa, t}\left(\frac{1}{n}\right) .
$$

(iv) For any disk $D$ in $H_{n-2}$ which meets $P(t)$ and any two dual disks $\tilde{D}_{1}, \widetilde{D}_{2}$, which meet $D$, we have

$$
M_{\tilde{D}_{1}}(z)-M_{\tilde{D}_{2}}(z)=O_{t}\left(\frac{1}{n^{3}}\right), \quad \forall z \in \tilde{D}
$$

Lemma 1.8. Let $t>0$, and normalize the packings $H_{n}$ and $H_{n}^{\prime}$ as in Corollary 1.7. There is a $\delta=\delta(\kappa, t)>0$ such that, for any $z_{0} \in P(t)$,

$$
\left|g_{n}(z)-M_{z_{0}}(z)\right| \leq C_{\kappa, t}\left|z-z_{0}\right|^{3}+\frac{C_{\kappa, t}}{n^{2}}, \quad \forall\left|z-z_{0}\right| \leq \delta .
$$

If $z$ is in the union of dual disks of $H_{n}$ and also satisfies $\left|z-z_{0}\right| \leq \delta$, then

$$
\left|g_{n}(z)-M_{z_{0}}(z)\right| \leq C_{\kappa, t}\left|z-z_{0}\right|^{3}+\frac{C_{\kappa, t}}{n^{3}}
$$

Proof. Let $\tilde{D}$ be a dual disk of $H_{n-2}$ which intersects $P(t)$ and let $D$ be a disk of $H_{n-2}$ which intersects $\tilde{D}$. On $\partial D, M_{\delta}$ is $O_{\kappa, t}\left(1 / n^{3}\right)$-close to $g_{n}$, and $g_{n}$ is $O_{\kappa, t}\left(1 / n^{2}\right)$ close to a similitude $S$ of $D$ onto $D^{\prime}$ (Corollary 1.7). Therefore the center of $D$ is moved by $M_{D}$ a distance $O_{\kappa, t}\left(1 / n^{2}\right)$. By symmetry in $\partial D$, which has radius $O_{\kappa}(1 / n)$, it follows that the pole of $M_{D}$ is at a minimum distance $C_{\kappa, t}>0$ from the center 
of $D$. Consequently there is a $\delta=\delta(\kappa, \mathfrak{t})>0$ such that, for $|z-\eta| \leq 2 \delta, M_{\tilde{D}}(z)$ is bounded away from $\infty$ and $(d / d z) M_{\tilde{D}}(z)$ is bounded away from 0 and $\infty$. Choose $\delta<t / 3$. (We remark for later reference that the second and third derivatives of $M_{D}$ are also bounded away from $\infty$ in this neighborhood of $\eta$.)

One consequence is that (1.12) holds trivially if $n$ is bounded. We may therefore assume $n$ is large ( $>6 t$ ) and $z_{0}$ is the center of some disk $D_{0}$ of $H_{n-2}$.

Given $z$ with $\left|z-z_{0}\right| \leq \delta$, construct an alternating sequence $D_{0}, \tilde{D}_{0}, D_{1}, \ldots, D_{m}$, $\tilde{D}_{m}$ of disks and dual disks such that adjacent disks intersect, each disk is at a distance $\leq 2 \delta$ from $z_{0}$ and hence in $P_{t / 3}, z \in \tilde{D}_{m} \cup \bar{D}_{m}$, and

$$
m \leq C_{\kappa} n\left|z-z_{0}\right|
$$

Since $z \in \tilde{D}_{m} \cup \bar{D}_{m}$ and $g_{n}=M_{\tilde{D}_{m}}$ in $\tilde{D}_{m}$, it follows from (1.9) that

$$
g_{n}(z)-M_{\tilde{D}_{m}}(z)=O_{\kappa, t}\left(\frac{1}{n^{2}}\right) .
$$

Thus the proof will be complete if we show that

$$
\left|M_{\tilde{D}_{m}}(z)-M_{\tilde{D}_{0}}(z)\right| \leq C_{\kappa, t}\left|z-z_{0}\right|^{3}
$$

By (1.11), for $0 \leq k \leq m-1$,

$$
M_{\tilde{D}_{k}}(\zeta)-M_{\tilde{D}_{k+1}}(\zeta)=O_{\kappa, t}\left(\frac{1}{n^{3}}\right), \quad \forall \zeta \in \bar{D}_{k}
$$

By the remark at the beginning of the proof concerning the boundedness of the derivatives, (1.17) implies

$$
M_{\tilde{D}_{k+1}}^{-1} \circ M_{\tilde{D}_{k}}(\zeta)-\zeta=O_{\kappa, t}\left(\frac{1}{n^{3}}\right), \quad \forall \zeta \in \bar{D}_{k} .
$$

Now $T_{k}=M_{\tilde{D}_{k+1}}^{-1} \circ M_{\tilde{D}_{k}}$ is a Möbius transformation which leaves $D_{k}$ invariant and is small on $\partial D_{k}$; we wish to estimate its magnitude at $z$, a distance

$$
\leq C_{\kappa}(m-k+1) / n
$$

from the center of $D_{k}$. For that purpose we make the following observation, the verification of which is left until the end of this proof.

Observation. Let $T$ be a Möbius transformation which leaves invariant a disk of radius $r$ centered at $\eta$. Suppose $\|T(z)-z\|_{|z-\eta|=r}<\varepsilon_{0}$ where $\varepsilon_{0}<r / 2$. Then $\|T(z)-z\|_{|z-\eta|=R}<12 \varepsilon_{0} R^{2} / r^{2}$ where $r<R<r^{2} / 2 \varepsilon_{0}$.

In order to apply this observation to $T_{k}=M_{\tilde{D}_{k+1}}^{-1} \circ M_{\tilde{D}_{k}}$ set $r=O_{\kappa}(1 / n)$, 
$\varepsilon_{0}=O_{\kappa, t}\left(1 / n^{3}\right), R \leq C_{\kappa}(m-k+1) / n$, and obtain

$$
\left|M_{\tilde{D}_{k+1}}^{-1} \circ M_{\tilde{D}_{k}}(z)-z\right|<C_{\kappa, t}\left(\frac{1}{n}\right)\left(\frac{m-k+1}{n}\right)^{2},
$$

or, by using the boundedness of the derivative again,

$$
\left|M_{\tilde{D}_{k}}(z)-M_{\tilde{D}_{k+1}}(z)\right|<C_{\kappa, t}\left(\frac{1}{n}\right)\left(\frac{m-k+1}{n}\right)^{2} .
$$

Summing (1.18) from $k=0$ to $m-1$ gives

$$
\left|M_{\tilde{D}_{m}}(z)-M_{\tilde{D}_{0}}(z)\right| \leq C_{\kappa, t}\left(\frac{1}{n^{3}}\right)\left(2^{2}+3^{2}+\cdots+(m+1)^{2}\right) \leq C_{\kappa, t}\left(\frac{m^{3}}{n^{3}}\right),
$$

since $1^{2}+2^{2}+\cdots+j^{2}=j(j+1)(2 j+1) / 6$. Since $m / n<C_{\kappa}\left|z-z_{0}\right|$ (see (1.14)), (1.19) implies (1.16) which completes the proof of (1.12).

If $z$ is also in the union of dual disks of $H_{n}$, then the right-hand side of (1.15) can be replaced by $O_{\kappa, t}\left(1 / n^{3}\right)$, and we obtain (1.13).

To verify the observation, assume $\eta=0$ and write $T$ in the form

$$
T(z)=\frac{r^{2} e^{i \theta}(z-\alpha)}{r^{2}-\bar{\alpha} z}
$$

Note that $|T(0)-0|=|\alpha| \leq \varepsilon_{0}$. From $|T(r)-r|<\varepsilon_{0}$ obtain

$$
\left|\mathrm{r}^{3}\left(e^{i \theta}-1\right)-r^{2}\left(e^{i \theta} \alpha-\bar{\alpha}\right)\right|<\varepsilon_{0}|r-\bar{\alpha}| r<2 \varepsilon_{0} r^{2},
$$

and therefore $\left|r^{3}\left(e^{i \theta}-1\right)\right|<2 \varepsilon_{0} r^{2}+r^{2}\left|e^{i \theta} \alpha-\bar{\alpha}\right|<4 \varepsilon_{0} r^{2}$. This shows that

$$
\left|e^{i \theta}-1\right|<\frac{4 \varepsilon_{0}}{r}
$$

Similarly, $T(z)-z=\left(r^{2} z\left(e^{i \theta}-1\right)-r^{2} e^{i \theta} \alpha-\bar{\alpha} z^{2}\right) /\left(r^{2}-\bar{\alpha} z\right)$. Now

$$
\left|r^{2}-\bar{\alpha} z\right| \geq r^{2}-|\alpha| R \geq \frac{r^{2}}{2}
$$

since $|\alpha| R \leq \varepsilon_{0} R \leq r^{2} / 2$. Using this and (1.20) we obtain

$$
\begin{aligned}
|T(z)-z| & \leq\left(\frac{2}{r^{2}}\right)\left|r^{2} z\left(e^{i \theta}-1\right)-r^{2} e^{i \theta} \alpha-\bar{\alpha} z^{2}\right| \\
& \leq\left(\frac{2}{r^{2}}\right)\left[r^{2} R\left(\frac{4 \varepsilon_{0}}{r}\right)+r^{2} \varepsilon_{0}+\varepsilon_{0} R^{2}\right] \\
& =8 \varepsilon_{0}\left(\frac{R}{r}\right)+2 \varepsilon_{0}+2 \varepsilon_{0}\left(\frac{R}{r}\right)^{2} \\
& \leq 12 \varepsilon_{0}\left(\frac{R}{r}\right)^{2} .
\end{aligned}
$$




\section{Global Theory}

We now consider the situation in [RS] of circle packing approximations to the Riemann mapping function. Let $\Omega$ be a bounded simply connected region in the plane, let $a$ and $b$ be given points of $\Omega$, and let $f$ be the Riemann map of $\Omega$ onto the unit disk $\mathbb{D}$ normalized by the two conditions: $f(a)=0$, and $f(b)$ lies on the positive real axis.

The circle packing approximations to $f$ are constructed as follows (see [RS] for complete details). Almost fill $\Omega$ with circles of radius $\varepsilon>0$ packed in the regular hexagonal circle packing pattern. Consider an isomorphic circle packing of $\mathbb{D}$ such that two circles of $\Omega$, one nearest $a$ and the other nearest $b$, are centered at the origin and on the positive real axis.

This correspondence of circles may be considered an approximate mapping $f_{\varepsilon}$ of $\Omega$ onto $\mathbb{D}$. We define the first derivative and second derivatives of $f_{\varepsilon}$ at $z$ as follows. Pick a dual circle closest to $z$, let $M_{z}$ be the Möbius transformation which maps it and the three distinguished points on it to the corresponding dual circle and three distinguished points in the packing of $\mathbb{D}$. Set $f_{\varepsilon}^{\prime}(z)=M_{z}^{\prime}(z), f_{\varepsilon}^{\prime \prime}(z)=M_{z}^{\prime \prime}(z)$. We shall see that $f_{\varepsilon}^{\prime}, f_{\varepsilon}^{\prime \prime}$ converge to $f^{\prime}, f^{\prime \prime}$. We also obtain improved estimates of the rate of convergence of $f_{\varepsilon}$ to $f$.

In order to make convenient use of the local theory in Section 1, let us redefine $f_{\varepsilon}$ so it will be an actual homeomorphism of an approximating subdomain $\Omega_{\varepsilon} \subset \Omega$ onto an approximating domain $\mathbb{D}_{\varepsilon} \subset \mathbb{D}$. For a nonboundary disk $D$ in the packing of $\Omega$, there is a maximal $H_{m}$ configuration of disks surrounding it. There is a corresponding $H_{m}^{\prime}$ configuration in $\mathbb{D}$. If $m \geq 2$, define $f_{\varepsilon}$ in $D$ and the six dual disks intersecting $D$ to be the mapping $g$ of Lemma 1.1 .

Lemma 2.1. Let $K \subset \subset \Omega$. There are constants $C_{K}>0$ and $\delta_{K}>0$ such that, for any $z_{0} \in K$,

$$
\left|f_{\varepsilon}(z)-\left[f_{\varepsilon}\left(z_{0}\right)+f_{\varepsilon}^{\prime}\left(z_{0}\right)\left(z-z_{0}\right)+\frac{1}{2} f_{\varepsilon}^{\prime \prime}\left(z_{0}\right)\left(z-z_{0}\right)^{2}\right]\right| \leq C_{K}\left|z-z_{0}\right|^{3}+C_{K} \varepsilon^{2}
$$

for $\left|z-z_{0}\right|<\delta_{K}$. If in addition $z$ is in the union of dual disks, then the right-hand side can be improved to $C_{K}\left|z-z_{0}\right|^{3}+C_{K} \varepsilon^{3}$.

Proof. If $z_{0} \in K$ and $D_{0}$ is an $\varepsilon$-disk in the packing of $\Omega$ nearest to $z_{0}$, then there is an $H_{n}$ configuration centered at $D_{0}$, where $n \geq \kappa / \varepsilon$ for $\kappa \equiv \operatorname{Dist}(K, \partial \Omega) / 4$. By Lemma 1.8 there is a $\delta_{K}>0$ such that $\left|f_{\varepsilon}(z)-M_{z_{0}}(z)\right|<C_{K}\left(\left|z-z_{0}\right|^{3}+\varepsilon^{2}\right)$ for $\left|z-z_{0}\right|<\delta_{K}$ (or $C_{K}\left(\left|z-z_{0}\right|^{3}+\varepsilon^{3}\right.$ ) if in addition $z$ is in the union of dual disks) where we write $C_{K}$ instead of $C_{\kappa}$. Thus we have

$$
\begin{aligned}
\mid f_{\varepsilon}(z)- & {\left[f_{\varepsilon}\left(z_{0}\right)+f_{\varepsilon}^{\prime}\left(z_{0}\right)\left(z-z_{0}\right)+\frac{1}{2} f_{\varepsilon}^{\prime \prime}\left(z_{0}\right)\left(z-z_{0}\right)^{2}\right] \mid } \\
\leq & \left|f_{\varepsilon}(z)-M_{z_{0}}(z)\right|+\left|M_{z_{0}}\left(z_{0}\right)-f_{\varepsilon}\left(z_{0}\right)\right| \\
& +\left|M_{z_{0}}(z)-M_{z_{0}}\left(z_{0}\right)-M_{z_{0}}^{\prime}\left(z_{0}\right)\left(z-z_{0}\right)-\frac{1}{2} M_{z_{0}}^{\prime \prime}\left(z_{0}\right)\left(z-z_{0}\right)^{2}\right| \\
\leq & C_{K}\left|z-z_{0}\right|^{3}+C_{K} \varepsilon^{2}+C_{K} \varepsilon^{2}+C_{K}\left|z-z_{0}\right|^{3},
\end{aligned}
$$

where the estimate on the last term rests on the remark on the boundedness of third derivatives in the proof of Lemma 1.8 . 
The next lemma is used to improve the estimate in [R2, Theorem 5] for the rate of convergence of $f_{n} \rightarrow f$.

Lemma 2.2. ${ }^{1} \quad$ Let $g(z)=z+a_{2} z^{2}+\cdots$ be a schlict function defined in the unit disk $\mathbb{D}$ and let $\varepsilon>0$. Then there is a constant $C$ depending only on $\varepsilon$ such that, for all measurable subsets $A \subset \mathbb{D}$, meas $(A) \leq C\left(\operatorname{meas}(g(A))^{(1 / 3)-\varepsilon}\right)$.

Proof. Let $1 / p=\left(\frac{1}{3}\right)-\varepsilon, 1 / q=\left(\frac{2}{3}\right)+\varepsilon$ so $(1 / p)+(1 / q)=1$. Then

$$
\begin{aligned}
\operatorname{meas}(A) & =\iint_{A}\left|g^{\prime}\right|^{2 / p}\left|g^{\prime}\right|^{-2 / p} d x d y \\
& \leq\left\{\iint_{A}\left(\left|g^{\prime}\right|^{2 / p}\right)^{p} d x d y\right\}^{1 / p}\left\{\iint_{A}\left(\left|g^{\prime}\right|^{-2 / p}\right)^{q} d x d y\right\}^{1 / q} .
\end{aligned}
$$

Now $2 q / p=1-3 \varepsilon /\left(\left(\frac{2}{3}\right)+\varepsilon\right)<1$. By the Koebe Distortion Theorem,

$$
\left|g^{\prime}(z)\right| \leq \frac{C}{(1-|z|)}
$$

so

$$
\iint_{A}\left|g^{\prime}\right|^{-2 q / p} d x d y \leq C \iint_{\mathbb{D}}(1-|z|)^{-2 q / p} d x d y \leq C .
$$

Therefore

$$
\operatorname{meas}(A) \leq C\left\{\iint_{A}\left(\left|g^{\prime}\right|^{2} d x d y\right\}^{1 / p}=C\left(\operatorname{meas}(g(A))^{(1 / 3)-\varepsilon}\right)\right.
$$

Lemma 2.3. Let $K \subset \subset \Omega$. Then there is a constant $C_{K}$ such that

$$
\left\|f_{\varepsilon}-f\right\|_{K} \leq C_{K} \varepsilon^{0.1428}
$$

Proof. We make use of the proof of Theorem 5 in [R2]. There we wrote $W_{\varepsilon}$ for the domain of $f_{\varepsilon}, D_{\varepsilon}$ for the image of $f_{\varepsilon}$, and we considered the Riemann mapping functions $G_{W_{\varepsilon}}: W_{\varepsilon} \rightarrow \mathbb{D}$ and $G_{D_{\varepsilon}}: D_{\varepsilon} \rightarrow \mathbb{D}$ which are normalized by the conditions

\footnotetext{
${ }^{1}$ Christian Pommerenke (oral communication) has shown that the exponent $\left(\frac{1}{3}\right)-\varepsilon$ can be improved to 0.405 ... by applying the proof given here in conjunction with a result of his (On the integral means of the derivative of a univalent function, J. London Math. Soc. (2) 32 (1985), 254-258), and could be replaced by 0.5 if the Brannan conjecture (J. E. Brannan, The integrability of the derivative in conformal mapping, J. London Math. Soc. (2) 18 (1978), 261-272) were true.
} 
$G_{W_{\varepsilon}}\left(z_{0}\right)=0, G_{W_{\varepsilon}}\left(z_{1}\right)>0$ and $G_{D_{\varepsilon}}(0)=0, G_{D_{\varepsilon}}\left(f_{\varepsilon}\left(z_{0}\right)\right)>0$. We then estimated each term on the right-hand side of

$$
\begin{aligned}
\left|f(z)-f_{\varepsilon}(z)\right| \leq & \left|f(z)-G_{W_{\varepsilon}}(z)\right|+\left|G_{W_{\varepsilon}}(z)-G_{D_{\varepsilon}} \circ f_{\varepsilon} \circ G_{W_{\varepsilon}}^{-1}\left(G_{W_{\varepsilon}}(z)\right)\right| \\
& +\left|G_{D_{\varepsilon}}\left(f_{\varepsilon}(z)\right)-f_{\varepsilon}(z)\right|
\end{aligned}
$$

(see (3.1) and (3.17) in [R2]). The first and last terms on the right were found to be bounded by $C_{K} \varepsilon^{1 / 2}$ and $C_{K} \varepsilon^{1 / 4}$, respectively. We now obtain an improved estimate of the middle term. Now $h \equiv G_{D_{\varepsilon}} \circ f_{\varepsilon} \circ G_{W_{\varepsilon}}^{-1}$ is a quasi-conformal self-map of $\mathbb{D}$; we estimate its bad area and then use the "Groetsch method" to compare $h$ with the identity map.

Let $\rho$ be a positive number which may depend on $\varepsilon$; we postpone an explicit choice of $\rho$ until the end of the proof. The bad area of $f_{\varepsilon}$ in $\Omega$ consists of two parts: the part in $K_{\rho} \equiv\{z \in \Omega$ : $\operatorname{dist}(z, \partial \Omega) \geq \rho\}$, call it $B_{1}$, and the part $B_{2}$ in $\Omega-K_{\rho}$. The bad area of $B_{1}$ lies inside disks of $\Omega(\varepsilon)$, and since each such disk is at a distance $\rho$ or more from $\partial \Omega$, it is the center of at least $[\rho / \varepsilon]$ generations of such disks. Therefore, by Lemma 1.1 , the bad area inside it is no greater than $\left(C /[\rho / \varepsilon]^{2}\right)\left(\pi \varepsilon^{2}\right)$. Since the area of $\Omega$ is bounded, the total bad area $B_{1}$ is no greater than $C \varepsilon^{2} / \rho^{2}$.

The bad area of $h \equiv G_{D_{\varepsilon}} \circ f_{\varepsilon} \circ G_{W_{\varepsilon}}^{-1}$ inside $\mathbb{D}$ is contained in the union of $G_{W_{\varepsilon}}\left(B_{1}\right)$ and the part $G_{W_{\varepsilon}}\left(B_{2}\right)$. Lemma 2.2 gives meas $\left(G_{W_{\varepsilon}}\left(B_{1}\right)\right) \leq C\left(\operatorname{meas}\left(B_{1}\right)\right)^{(1 / 3)-\beta}(\beta>0$ but arbitrary). Since we have meas $\left(B_{1}\right) \leq C \varepsilon^{2} / \rho^{2}$, we conclude that

$$
\operatorname{meas}\left(G_{W_{\varepsilon}}\left(B_{1}\right)\right) \leq C\left(\frac{\varepsilon^{2}}{\rho^{2}}\right)^{(1 / 3)-\beta}
$$

It is well known (see [W] for references) that $G_{W_{\varepsilon}}\left(B_{2}\right)$ lies in $\left\{1-\rho^{1 / 2} \leq|z| \leq 1\right\}$. Thus

$$
\operatorname{meas}\left(G_{W_{\varepsilon}}\left(B_{2}\right)\right) \leq C \rho^{1 / 2}
$$

We now determine $\rho$ by setting $\rho=\varepsilon^{4 / 7}$; this transforms (2.2) and (2.3) into

$$
\text { bad area of } h \leq C \varepsilon^{2 / 7-\beta} \approx C \varepsilon^{0.2857} \text {, }
$$

where $\beta$ is an arbitrary preassigned positive number.

By the Grötsch argument for conformal moduli (see the proof of Lemma 1.3 above) we can conclude that $|h(w)-w| \leq C\left(\varepsilon^{0.2857}\right)^{1 / 2}=C \varepsilon^{0.14285}$ for all $w \in \mathbb{D}$. We now return to (2.1) and obtain

$$
\left|f(z)-f_{\varepsilon}(z)\right| \leq C_{K}\left(\varepsilon^{1 / 2}+\varepsilon^{1 / 4}+\varepsilon^{0.1428}\right) \leq C_{K} \varepsilon^{0.1428}
$$

which completes the proof of Lemma 2.3. 
Theorem 2.4. Let $f_{\varepsilon}$ be the circle packing approximation to the Riemann mapping function $f: \Omega \rightarrow \mathbb{D}$. Let $f_{\varepsilon}^{\prime}, f_{\varepsilon}^{\prime \prime}$ be the first and second derivatives of $f_{\varepsilon}$ as in Lemma 2.1. Let $K \subset \subset \Omega$. There exists a constant $C_{K}$ such that, for all $z$ in $K$,

$$
\begin{gathered}
\left|f_{\varepsilon}(z)-f(z)\right| \leq C_{K} \varepsilon^{0.1428} \\
\left|f_{\varepsilon}^{(1)}(z)-f^{\prime}(z)\right| \leq C_{K} \varepsilon^{0.1428 / 2} \\
\left|f_{\varepsilon}^{(2)}(z)-f^{\prime \prime}(z)\right| \leq C_{K} \varepsilon^{0.1428 / 4}
\end{gathered}
$$

Proof. The first inequality is Lemma 2.3. Since $f$ is analytic in $\Omega$ we have, for all $z, \zeta_{0}$ in $K$,

$$
\left|f(z)-f\left(\zeta_{0}\right)-f^{\prime}\left(\zeta_{0}\right)\left(z-\zeta_{0}\right)\right| \leq C_{K}\left|z-\zeta_{0}\right|^{2}
$$

By Theorem 2.1,

$$
\left|f_{\varepsilon}(z)-f_{\varepsilon}\left(\zeta_{0}\right)-f_{\varepsilon}^{\prime}\left(\zeta_{0}\right)\left(z-\zeta_{0}\right)\right| \leq C_{K}\left(\left|z-\zeta_{0}\right|^{2}+\varepsilon^{2}\right)
$$

Combining these with (2.5) we obtain

$$
\left|f_{\varepsilon}^{\prime}\left(\zeta_{0}\right)\left(z-\zeta_{0}\right)-f^{\prime}\left(\zeta_{0}\right)\left(z-\zeta_{0}\right)\right| \leq C_{K}\left(\left|z-\zeta_{0}\right|^{2}+\varepsilon^{0.1428}\right)
$$

Hence

$$
\left|f_{\varepsilon}^{\prime}\left(\zeta_{0}\right)-f^{\prime}\left(\zeta_{0}\right)\right| \leq C_{K} \inf \left\{\left|z-\zeta_{0}\right|+\frac{\varepsilon^{0.1428}}{\left|z-\zeta_{0}\right|}: z \in K\right\}
$$

By choosing $z \in K$ to satisfy $\left|z-\zeta_{0}\right|=\varepsilon^{0.1428 / 2}$ we obtain (2.6). Similarly, (2.7) follows from (2.5), (2.6), and Theorem 2.1.

\section{References}

[Ah] D. Aharanov, The hexagonal packing lemma and discrete potential theory, Canad. Math. Bull. $33(1990), 247-252$

[H] Z.-X. He, An estimate for hexagonal circle packings, J. Differential Geom. 33 (1991), 395-412.

[HR] Z.-X. He and B. Rodin, Convergence of circle packings of finite valence to Riemann mappings, Preprint.

[MR] A. Marden and B. Rodin, On Thurston's Formulation and Proof of Andreev's Theorem, Lecture Notes in Mathematics, Vol. 1435, Springer-Verlag, Berlin, (1990), pp. 103-115.

[R1] B. Rodin, Schwarz's lemma for circle packings, I, Invent. Math. 89 (1987), 271-289.

[R2] B. Rodin, Schwarz's lemma for circle packings, II, J. Differential Geom. 30 (1989), $539-554$.

[RS] B. Rodin and D. Sullivan, The convergence of circle packings to the Riemann mapping, J. Differential Geom. 26 (1987), 349-360.

[S1] O. Schramm, Rigidity of infinite (circle) packings, J. Amer. Math. Soc. 4 (1991), 127-149. 
[S2] O. Schramm, Existence and uniqueness of packings with specified combinatorics, Israel $J$. Math. 73 (1991), 321-341.

[T] W. P. Thurston, The finite Riemann mapping theorem, invited address, International Symposium in Celebration of the Proof of the Bieberbach Conjecture, Purdue University, March 1985.

[W] S. E. Warschawski, On the degree of variation in conformal mapping of variable regions, Trans. Amer. Math. Soc. 69 (1950), 335-356.

Received May 4, 1992. 